\title{
BEAM DIAGNOSTIC SYSTEM FOR HIGH INTENSITY PROTON LINAC AT KAERI
}

\author{
S. J. Park, J. H. Park, Y. S. Bae, W. H. Hwang, J. Y. Huang, and S. H. Nam, \\ Pohang Accelerator Laboratory, POSTECH, Pohang, Korea \\ and \\ Y. S. Cho, J. M. Han, S. H. Han, B. H. Choi \\ Proton Engineering Frontier Project, KAERI
}

\begin{abstract}
A $100-\mathrm{MeV}$ high-intensity $(20 \mathrm{~mA} \mathrm{CW})$ proton linac is being built in the KAERI (Korea Atomic Energy Reserach Institute), Korea. Beam diagnostic system for the machine require to include BCMs (Beam Current Monitors), BPPMs (Beam Position and Phase Monitors), and other devices. BCMs are of special importance for high-intensity proton linacs, since the control of beam loss is critical. They should have high stability and resolution better than 5E-4. BPPMs are for measuring beam position and phase (w.r.t RF) simultaneously, that are crucial for the successful commissioning and operation of the accelerator. All diagnostic devices are required to operate both in $\mathrm{CW}$ and pulsed (several ms) modes. Pulsed mode operation is useful when machine tuning and commissioning. In this article, we report the developmental status of beam diagnostic devices for the KOMAC accelerator, with detailed description on the BPPM PU design.
\end{abstract}

\section{INTRODUCTION}

The KOMAC (KOrea Multi-purpose Accelerator Complex) is one of HPPAs (High-Power Proton Accelerators) which are under construction at the KAERI (Korea Atomic Energy Research Institute), Korea. It aims to generate $100-\mathrm{MeV}$ proton beams at the beam current of $20 \mathrm{~mA}$, CW. Refer to Table 1 for the major beam parameters of the KOMAC accelerator.

Table 1: Beam parameters of KOMAC accelerator

\begin{tabular}{|c|c|c|c|}
\hline \multirow{2}{*}{} & \multicolumn{2}{|c|}{ Operation Modes } & \multirow{2}{*}{ Remark } \\
\cline { 2 - 3 } & CW & Pulse & \\
\hline Beam Energy & \multicolumn{2}{|c|}{$100 \mathrm{MeV}$} & \\
\hline$\beta$ & \multicolumn{2}{|c|}{0.43} & \\
\hline$\gamma$ & \multicolumn{2}{|c|}{1.107} & \\
\hline Beam Current & $\begin{array}{c}20 \mathrm{~mA} \\
\text { max. }\end{array}$ & $\begin{array}{c}20 \mathrm{~mA} \\
\text { peak }\end{array}$ & \\
\hline Pulse Width & NA & $2 \mathrm{~ms}$ & \\
\hline Bunch Length & \multicolumn{2}{|c|}{$160 \mathrm{ps}$} & $\begin{array}{c}\text { PARMILA } \\
\text { Simulation }\end{array}$ \\
\hline $\begin{array}{c}\text { Bunching } \\
\text { Frequency }\end{array}$ & \multicolumn{2}{|c|}{$350 \mathrm{MHz}$} & \\
\hline
\end{tabular}

The beginning parts of HPPAs includes ion sources, RFQs and DTLs which are complex and have tight installation spaces. This is especially true for diagnostic devices, and their design and installation often become designer's "nightmare." Meanwhile, diagnostics including BPMs and CTs are very important for successful commissioning and operation of the accelerators. Hence, their implementation should be considered from the early stage of the accelerator design. Successful compromise between the two conflicting side is possible when the diagnostic devices can be made compact without sacrificing their performances. Compact devices are easy to handle, economical, and generally have better highfrequency characteristics. Modern beam diagnostic devices are far more compact than their ancestors. For example, some CTs from the Bergoz Instrumentations are now integrated with $\mathrm{CF}$ flanges and their total length (axial) can be made as small as $30 \mathrm{~mm}$. Majority of BPM PUs (Pick-Ups) for proton accelerators are still striplines that yield well defined response even for low-intensity beams. But their sizes are still too big to be installed in the narrow front-ends the accelerator. For example, the axial length of the striplines for the SNS (Spallation Neutron Source, USA) linac exceeds $100 \mathrm{~mm}$, even if they are installed in the vacuum. Button-type capacitive PUs have been simple and reliable, but their application to the proton machines has been limited because of their insufficient response to low-intensity beams. Modern HPPAs such as the KOMAC are designed to have very high beam intensities, so that even the buttons could generate enough signals for precision beam position measurements. In this regards, we have chosen the button-type PU for use in the KOMAC accelerator. One of the disadvantages of the buttons is that, it is difficult to predict the PU sensitivity using analytic formulas. In fact, the PU sensitivity for low-beta beams can not be practically determined even by experimental methods, due to the difficulty of simulating electromagnetic fields from the low-beta beams. We have decided to utilize the computer code for determining the sensitivity of the button-type PU. We have chosen the MAGIC code which is a kind of the PIC (Particle-In-Cell) code and can treat the particle and electromagnetic system in the full three dimensional manner. 


\section{DESIGN OF BPPM PU}

The theoretical estimation of the sensitivity of stripline PU was established by R. E. Shafer ${ }^{[1]}$ It is basically $2 \mathrm{D}$ theory and can not be used for designing the button-type PUs which are 3D features. There are commercial electromagnetic codes that can simulate 3D geometries, including the MAFIA T3. But the MAFIA T3 can handle only ultra-relativistic particles and can not be applied to low-beta beams. (The port boundary of the MAFIA T3 can accept only TEM mode whose phase velocity is equal to that of the light. And low-beta beams generate fields that are not in the simple TEM mode.) Limitations of the Shafer's theory and the MAFIA code have led us to consider on using the MAGIC code. It is versatile and can handle arbitrary combinations of particle beams and electromagnetic structures.

Fig. 1 shows the 3D model of the button PU for the MAGIC simulation and its cross-sectional view in the transverse plane. In the left figure of Fig. 1, proton bunches travel left-to-right direction. Beam signals are coupled to the four buttons that are installed around the circumference of the beam pipe. Buttons are connected to 50-ohm coaxial lines whose dimensions are different from those of usual fabrication. This simplified the simulation geometry without sacrificing reliability of the simulation.
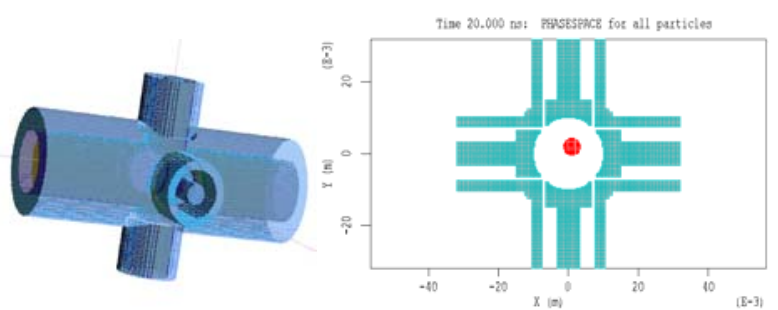

Figure 1: Modelling of button-type PU for MAGIC simulation. Left figure: 3D model, Right figure: Crosssectional view. Dot inside beam pipe in right figure indicates proton beam. Diameter of beam pipe is $20 \mathrm{~mm}$.

Default beam parameters used in the simulation are shown in Table 2. The left figure of Fig. 2 is the voltage waveform developed between the inner and outer conductors of the coaxial line. As expected, they are considerably longer than the beam bunch length, which is due to the axial extension of fields from the low-beta beams. ( $\beta=0.08$ for $\mathrm{E}=3 \mathrm{MeV}$ ) With increasing the beam energy, beam fields concentrate in the transverse plane, and the pulse widths of signals coupled to buttons are shortened approaching that of charge distribution in the beam. This is shown in the right figure of Fig. 2 which is the signal waveform for the beam energy of $100 \mathrm{MeV}$.

Table 2: Default beam parameters used in MAGIC simulation.

\begin{tabular}{cc}
\hline Beam Energy & $\mathbf{3 ~ M e V}$ \\
\hline Avera ge (Peak) Beam Current & $20(570) \mathrm{mA}$ \\
\hline B unching Frequency & $350 \mathrm{MHz}$ \\
\hline Bunch Length & $43 \mathrm{ps} \mathrm{rms}$ \\
\hline
\end{tabular}

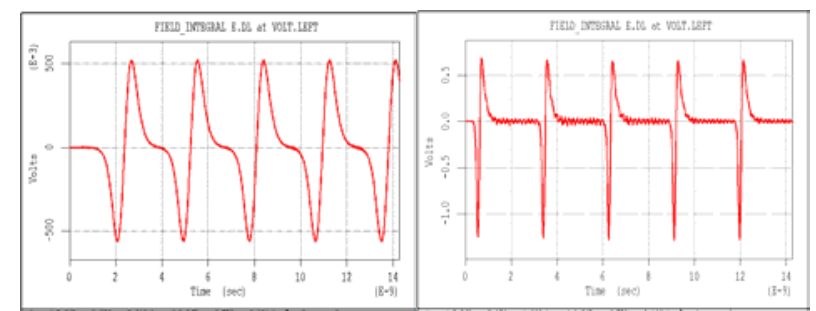

Figure 2: Waveforms of voltage signal developed at the end of one of coaxial lines for beam energies of $3 \mathrm{MeV}$ (left) and $100 \mathrm{MeV}$ (right).

The dependence of the PU sensitivity on the beam energy was simulated with result shown in Fig. 3. As expected from the Shafer's theory, the sensitivity increases dramatically with decreasing the beam energy. It also increases with increasing frequency at low energies but converge to the same value at high energies.



Figure 3: Dependence of PU sensitivity on beam energy.

Sensitivity of the PU to beam position change was simulated by moving the beam in the transverse plane in the step of $1 \mathrm{~mm}$. The sensitivity map was obtained by plotting the delta-over-sum values of band-pass filtered voltage signals appearing at the ends of the coaxial lines. The center frequencies and bandwidth of the band-pass filter were 350 or $700 \mathrm{MHz}$ and $20 \mathrm{MHz}$ respectively. See Fig. 4 for the sensitivity maps for the frequencies of 350 and $700 \mathrm{MHz}$.
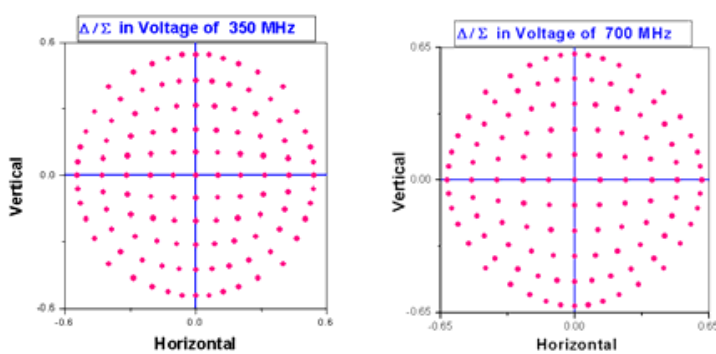

Figure 4: Sensitivity map of button PU at the frequencies of 350 and $700 \mathrm{MHz}$. Beam pipe diameter $=20 \mathrm{~mm}$.

Button diameter $=12 \mathrm{~mm}$. Beam energy and beta are 3 $\mathrm{MeV}$ and 0.08 respectively.

Note that the sensitivity for $700 \mathrm{MHz}$ was about $7.5 \%$ larger than that of the $350 \mathrm{MHz}$. 
In order to confirm the validity of the MAGIC simulation, we have simulated the BPM PU of the PLS (Pohang Light Source). The PU consists of four buttons with $9.5-\mathrm{mm}$ diameter installed in vacuum chamber that has diamond-like cross section. Table 3 compares some of simulation results with experimental measurements. Beam parameters used in the simulation are; Beam energy $=2.5 \mathrm{GeV}$, Average (Peak) current $=200$ (1600) $\mathrm{mA}$, Bunching frequency $=500 \mathrm{MHz}$, Bunch length $=26$ ps. The measured signal amplitude may contain substantial errors due to our rough estimation of the cable attenuation for the wide-band pulses. Simulated sensitivity values needs further refinement with finer meshes.

Table 3: Comparison between MAGIC simulation and experimental measurement results of PLS BPM PU.

\begin{tabular}{|c|c|c|c|}
\hline \multicolumn{2}{|c|}{} & Simulation & Measurement \\
\hline \multicolumn{2}{|c|}{ Signal Amplitude (V) } & 5.5 & 3 \\
\hline $\begin{array}{c}\text { On-center } \\
\text { Sensitivity (\%/mm) }\end{array}$ & $\mathrm{X}$ & 6.6 & 6.15 \\
\cline { 2 - 4 } & $\mathrm{Y}$ & 7.7 & 6.13 \\
\hline
\end{tabular}

The fabrication of the PU that consists of buttons, coaxial feedthroughs, and vacuum chamber is in progress at the Hitachi Electronics in Japan. A sample PU that is composed of one button + feedthrough and vacuum chamber will be fabricated by the company and its electrical performance will be tested in the Pohang Accelerator Laboratory.

\section{BPPM ELECTRONICS}

The signal processing electronics should provide both $\mathrm{CW}$ and pulsed measurement of beam position and phase for the beam current ranging from 0.2 to $20 \mathrm{~mA}(40-\mathrm{dB}$ dynamic range). Resolution and stability are required to be better than $50 \mu \mathrm{m}$. For this, the LR (Log-Ratio) electronics would be the best choice since it has large acquisition bandwidth $(>5 \mathrm{MHz})$ and reasonable dynamic range $(50 \mathrm{~dB}) \cdot{ }^{[2]}$ Resolution performance is rather marginal $(\sim 50 \mu \mathrm{m}$ at $20-\mathrm{mm} \varphi$ Beam pipe) but can be improved by the averaging multiple samples. Commercial LR electronics is already available from Bergoz Instrumentation at reasonable price. Phase measurement circuit could be fabricated in house, or custom-ordered from the company to make it integrated into the existing LR electronics avoiding the necessity of expensive connectors and cables.

\section{BCM DEVELOPMENT}

Efforts for developing BCM hardware will be saved by collaborating with companies including the Bergoz Instrumentation. In order to make BCMs highly stable and immune to bunch-length changes, it is recommended to limit the measurement bandwidth by tuning the current monitor to a specific frequency among various spectral contents in the beams. This can be realized by providing small capacitance, $\mathrm{C}$ in the winding of high-permeability core, which will oscillate at the frequency determined by the formula $f=1 / 2 \pi \sqrt{L C}$ where, $\mathrm{L}$ is the leakage inductance of the winding. ${ }^{[3]}$ By adjusting the value of $C$, the oscillation can be tuned to a specific harmonic (e.g., $350 \mathrm{MHz}$ ) among the beam spectrum. The oscillation should be made low $Q$ to avoid excessive or erratic responses. Prototype BCMs based on the above operating principle (Tuned-CT) are under fabrication in the company and their performance will be tested at the KOMAC accelerator.

\section{CONCULSION}

We conclude this article with the following summarizing remarks:

1. A button-type PU for use in the KOMAC (Korea Multi-purpose Accelerator Complex) accelerator was designed utilizing the MAGIC code.

2. Dependence of the PU sensitivity on the beam energy and the frequency well corresponded to the theoretical predictions.

3. Simulation results of the PLS (Pohang Light Source) electron BPM PU reasonably agreed to the experimental measurements.

4. "Tuned-CT" for measuring beam currents with high stability (5E-4) is under development with the collaboration with the Bergoz Instrumentation.

\section{ACKNOWLEDGEMENTS}

The authors would like to thank to Julien Bergoz, Klaus Unser in the Bergoz Instrumentation and Jim Hinkson in the GMW Associates for their numerous and enthusiastic discussions. This work was supported by the Ministry of Science and Technology, Korea.

\section{REFERENCES}

[1] R. E. Shafer, "Sensitivity of PUs for low beta beams," Proc. of the BIW93.

[2] A. Kalinin, "The Log-Ratio Beam Position Monitor," Proc. Of the BIW 2002, Upton, New York, 2002, p.384.

[3] Private communication with Bergoz Instrumentation. 